\title{
A Scalable Role Mining Approach for Large Organizations
}

\author{
Masoumeh Abolfathi \\ masoumeh.abolfathi@ucdenver.edu \\ University of Colorado, Denver \\ Denver, Colorado, USA \\ Haadi Jafarian \\ haadi.jafarian@ucdenver.edu \\ University of Colorado, Denver \\ Denver, Colorado, USA
}

\author{
Zohreh Raghebi \\ zohreh.raghebi@ucdenver.edu \\ University of Colorado, Denver \\ Denver, Colorado, USA \\ Farnoush Banaei-Kashani \\ farnoush.banaei-kashani@ucdenver.edu \\ University of Colorado, Denver \\ Denver, Colorado, USA
}

\begin{abstract}
Role-based access control (RBAC) model has gained significant attention in cybersecurity in recent years. RBAC restricts system access only to authorized users based on the roles and regulations within an organization. The flexibility and usability of this model have encouraged organizations to migrate from traditional discretionary access control (DAC) models to RBAC. However, this transition requires accomplishing a very challenging task called role mining in which users' roles are generated from the existing access control lists. Although various approaches have been proposed to address this NP-complete problem in the literature, they suffer either from low scalability such that their execution time increases exponentially with the input size, or they rely on fast heuristics with low optimality that generate too many roles. In this paper, we introduce a highly scalable yet optimal approach to tackle the role mining problem. To this end, we utilize a non-negative rank reduced matrix decomposition method to decompose a large-scale user-permission assignment into two constitutive components, i.e. the user-role and role-permission assignments. Then, we apply a thresholding technique to convert real-valued components into binary-valued factors. We employ various access control configurations and demonstrate that our proposed model is able to effectively discover the latent relationship behind the user-permission data even with large datasets.
\end{abstract}

\section{CCS CONCEPTS}

- Computer systems organization $\rightarrow$ Embedded systems; Redundancy; Robotics; • Networks $\rightarrow$ Network reliability.

\section{KEYWORDS}

Access Control; RBAC; Role Mining; Non-Negative Matrix Factorization

\footnotetext{
Permission to make digital or hard copies of all or part of this work for personal or classroom use is granted without fee provided that copies are not made or distributed for profit or commercial advantage and that copies bear this notice and the full citation on the first page. Copyrights for components of this work owned by others than ACM must be honored. Abstracting with credit is permitted. To copy otherwise, or republish, to post on servers or to redistribute to lists, requires prior specific permission and/or a fee. Request permissions from permissions@acm.org.

IWSPA'21, April 28, 2021, Virtual Event, USA

(C) 2021 Association for Computing Machinery.

ACM ISBN 978-1-4503-8320-2/21/04 ..\$15.00

https://doi.org/10.1145/3445970.3451154
}

\section{ACM Reference Format:}

Masoumeh Abolfathi, Zohreh Raghebi, Haadi Jafarian, and Farnoush BanaeiKashani. 2021. A Scalable Role Mining Approach for Large Organizations. In Proceedings of the 2021 ACM International Workshop on Security and Privacy Analytics (IWSPA'21), April 28, 2021, Virtual Event, USA. ACM, New York, NY, USA, 10 pages. https://doi.org/10.1145/3445970.3451154

\section{INTRODUCTION}

Unauthorized access to systems, data, and resources is a fundamental concern in commercial security. Organizations use access control models to define users' access rights or permissions. One typical model is the discretionary access control (DAC) where permissions are individually assigned to users. However, individual assignment of permissions is daunting, hard-to-manage, and unscalable, especially for a large or even medium-sized organization. The Role-based Access Control (RBAC) model is commonly used in commercial, governmental, and research sectors. In RBAC, users' access rights are not assigned individually, but rather are associated with roles. Roles are created for various job functions in an organization, and users are assigned to appropriate roles based on their responsibilities and qualifications. According to a report by IBM [5], RBAC facilitates security administration through group management capabilities. With RBAC, security is managed at a level that corresponds closely to the organization's structure. Therefore, many commercially successful access control systems implement RBAC. For successful deployment of RBAC in an organization, it is critically important to define a proper set of roles capable of capturing business processes within the organization. While proper identification and management of roles are more straightforward when roles are defined from scratch, in many cases, organizations have to transition to RBAC from an existing DAC-based model, where permissions are individually assigned to users. In such circumstances, the roles must be defined based on existing user-permission assignments. The role mining problem is a process in which roles are identified from the existing user-permission matrices. The goal of this process is to extract an optimal (usually minimum) set of roles from an input user-permission assignment matrix. This is an NP-complete problem [33], which is difficult to solve optimally, especially for large- or even medium-sized organizations, where the number of users, permissions, and candidate roles are high.

Researchers have proposed different approaches in the literature for role mining, using different methodologies and optimality metrics $[10,11,19,27,31,33,34]$. However, existing approaches either rely on heuristics that solve the NP-complete role mining problem 
for large input sizes in a scalable but non-optimal manner (resulting in the generation of too many roles) [1], or provide optimal or nearoptimal solutions which are only suited for small organizations and suffer from lack of scalability [11]. Even for scalable approaches, none of the existing methods have solved the role mining problem for organization sizes under our consideration.

In this paper, we propose a scalable and optimal role mining technique based on Non-Negative Matrix Factorization (NMF) [17]. Our proposed method performs accurate role mining for organizations with thousands of users and permissions. To discover the underlying semantic structure in user-permission data, we use an efficient matrix decomposition approach to derive user-role and role-permission relations with optimal decomposition rank.

In our approach, for optimal and scalable role mining, we decompose the user-permission binary matrix into two binary user-role and role-permission matrices. To address the scalability, we take advantage of the dimensionality reduction concept that is a feature of matrix decomposition. We use the NMF dimensionality reduction approach, which is based on the iterative learning algorithm. Due to iterative optimization, NMF often converges early despite the large size of the input [8]. Moreover, NMF optimality in recreating the factorized matrices has been proved $[3,4]$.

The security-sensitive nature of role mining aggravates the existing challenges of using matrix decomposition for any large machine learning task. These challenges include difficulties in determining the rank of the decomposition, converting a real-valued matrix into a binary matrix, and handling the risk of permission over- and under-assignments. In this paper, we propose techniques to alleviate these challenges and experimentally evaluate our approach. The proposed method is aimed at outputting a flat RBAC state that divides user-permission into two user-role and role-permission factors, and consists of the following steps:

- First, a large input user-permission matrix is decomposed into two low-rank factors that can be interpreted as user-role and role-permission assignments. This is performed via NMF [17]. Also, we obtain the optimal decomposition rank using the Elbow method [14].

- Second, real-valued factors from the previous step are converted into binary factors. This is because, for the role mining problem, the binary factors (user-role and role-permission) are needed rather than real-valued factors. To this end, we employ the thresholding method.

- In many cases, the proposed approach covers $100 \%$ of the user-permission assignment. In some cases, we cannot reconstruct up to $4 \%$ of the input data, all of which are underassignments of permissions. Thus, we propose a heuristic algorithm to cover these corner cases and to mitigate the possible security and usability impacts. Using this algorithm, we cover all under-assignments by refining the permissions for each user.

To evaluate the proposed approach, we run several experiments on five different datasets, including small (two datasets), medium (one dataset), and large (two datasets). In terms of user-permission coverage, the accuracy of our model is determined by calculating how much the two new binary factors, i.e., the user-role and role-permission assignments, can cover the original input userpermission assignment. Remarkably, our proposed method achieves $100 \%$ coverage on the small datasets. It also achieves $96 \%$ to $99 \%$ accuracy on larger datasets. Importantly, the $4 \%$ loss of accuracy can be eventually recovered by the proposed greedy prune-and-refine algorithm.

We define an optimal solution as one that yields the same $U P$ matrix with a minimum number of roles. In terms of optimality, our approach generates optimal solutions in most cases and provides a near-optimal solution for large-scale inputs. More specifically, the number of generated roles is often close to the number of original roles, which are used to construct the original user-permission matrix. Of note, our role mining approach on a highly large-scale input matrix with 400 million user-permission assignments creates only $10.87 \%$ increase in the number of generated roles, which is still acceptable.

The rest of this paper is organized as follows. In Section 2, we review the preliminaries and background knowledge needed to establish an understanding of the problem domain. We then discuss the related work and our proposed role mining approach in Sections 3 and 4, respectively. Experimental results are discussed in Section 5 , followed by conclusion and future research directions in Section 6.

\section{BACKGROUND AND PRELIMINARIES}

In this section, we review the notions of basic RBAC, matrix decomposition, and non-negative matrix factorization.

\subsection{Overview of RBAC Definition and Terminology}

In RBAC, a "role" is defined as a set of permissions on resources. New permissions can be granted to or revoked from roles as needed. A permission is an access right to an object in the system. The RBAC model is based on two key relations, i.e. the user to role (UR) and role to permission (RP) assignments. Both are many-to-many relations. A user can have many roles, and a role can be assigned to many users. Likewise, a role can include many permissions, and the same permission can be granted to many roles. Finally, it is a user who uses permissions. The placement of a role as mediator to enable a user to exercise permission provides more simplification on access control management than does directly on assigning users to permissions [20].

The following definitions formalize the above discussion.

- $U, R, P$ (users, roles, and permissions)

- $U R \subset U \times R$ : a many-to-many user to role assignment relation

- $R P \subset R \times P$ : a many-to-many role to permission assignment relation

- $U P \subset U \times P$ : a many-to-many users to permission assignment relation

- $R \in \mathcal{P}(P)-\emptyset$ (where $\mathcal{P}$ is power set)

\subsection{Matrix Decomposition}

In this work, we propose the use of approximate matrix decomposition to decompose the user-permission matrix into its constituent parts; namely, user-role and role-permission matrices (Figure 1). 
Matrix decomposition (also called matrix factorization) is a fundamental machine learning technique that factorizes an input matrix into a product of matrices. Depending on the constraints utilized, the resulting factors can have different representational properties. Restrictions include making matrices to be non-negative, binary, or all rows sum to 1.0 , etc. In this work, given $n$ users and $m$ permissions, a binary matrix $X_{n \times m}$ is decomposed into two matrices $A_{n \times k}$ and $B_{m \times k}$ such that $X \approx A \times B$. In other words, the distance between $X$ and $A B^{\top}$ denoted by $D\left(X, A B^{\top}\right)$ is minimized.

$$
\text { minimize }\left\|X-A B^{\top}\right\|
$$

In the matrix decomposition, when $X$ is decomposed to $A$ and $B$, the rank of $A$ and $B$ is typically $k$, i.e. $A \in \mathbb{R}^{n \times k}$ and $B \in \mathbb{R}^{m \times k}$. In the following section, we describe the NMF model and explain how it can be interpreted in the context of RBAC.

2.2.1 Non-Negative Matrix Factorization. NMF technique has gained practical significance due to its powerful interpretability and close relationship with the clustering methods. The sparseness of the data is another important aspect that is managed effectively by NMF. Moreover, the NMF technique has caught the attention of researchers due to its computational advantages. In the following, we discuss the striking properties of NMF that make it well-suited for the analysis of large-scale access control data.

- It is essential to capture relationships among users and permissions. In terms of non-negativity property, NMF is superior to other dimensionality reduction approaches $[16,17$, 28]. NMF makes it easy to interpret decomposed matrices, especially in the context of RBAC.

- The stochastic nature of the NMF algorithm enables it to run the steps of the algorithm concurrently. Therefore, the speed and efficiency would be substantially increased.

Based on the above discussion, advantages in computational efficiency and reconstruction quality makes it pertinent to consider the NMF technique as an applicable approach for role mining in large-scale organizations.

Non-negative matrix factorization is a classic clustering method designed to produce factors of a matrix. This is accomplished via a decomposition of the given matrix $G$ into two matrices with nonnegative entries, $G \approx W H$, where $W$ has size $n \times r$, and $H$ has size $r \times m$. Usually, the rank $r$ of the factorization is chosen so that $(n+$ $m) \times r<n m$, i.e. $r$ is less than $n$ and $m$. If the basis vectors discover the structure that is latent in the data, a good approximation can be achieved. In order to conduct NMF and quantify the quality of the approximation, we need to describe an objective function. Such an objective function can be defined using some measure of the distance between $G$ and the product $W H$. Examples of such measures include Euclidean distance as shown in Equation 2.

$$
\text { minimize }\|G-W H\|_{2}
$$

Computing NMF is an NP-hard problem, so the aim would be for an acceptable local minima. An implementation of the NMF method consists of iterative update rules for $W$ and $H$ matrices as given in the following equations:

$$
W_{i k} \leftarrow W_{i k} \sum_{j} \frac{G_{i j}}{(W H)_{i j}} H_{k j}
$$

and

$$
H_{k j} \leftarrow H_{k j} \sum_{i} W_{i k} \frac{G_{i j}}{(W H)_{i j}}
$$

Starting with random initial values for $W$ and $H$, the algorithm simultaneously updates these two matrices via multiplicative rules until convergence to a local minimum is achieved [3]. Algorithm 1 describes NMF in more details.

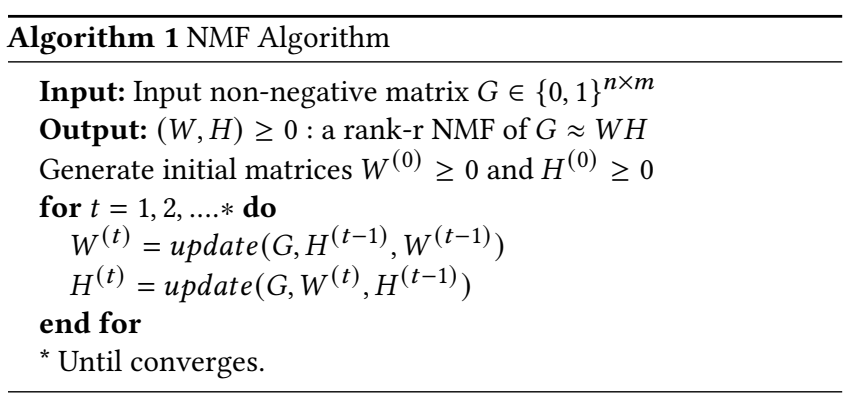

\section{RELATED WORK}

\subsection{Role Mining}

To address the role mining problem, Vaidya et al. propose the CompleteMiner (CM) method, which utilizes the concept of subset enumeration [34]. The authors preprocess the original data in phase 1 of the algorithm. Roles are then initialized from all possible existing permission combinations because each role is defined as a set of permissions. Next, the set InitRoles is generated by considering the users who have the same permissions as a unique role set. In phase 2, the CM enumerates all intersection between sets in InitRoles and adds them to the existing sets in InitRoles. Although the computational complexity of this algorithm is exponential, the authors made a notable improvement in their proposed algorithm by intersecting only pairs of InitRoles. The new method, which is called FastMiner [34] is a heuristic approach that finds only the roles that are maximally common to any two users. By doing so, the computational complexity of the algorithm reduces to $O\left(n^{2} m\right)$. However, this approach ignores some significant roles.

$\mathrm{Lu}$ et al. propose a bottom-up role engineering approach based on matrix decomposition and graph optimization [19]. In this model, matrix and graph are used for the representation of user-role permission assignments. Graph optimization is then utilized for finding optimal role hierarchies, thus reducing the cost of administration. The contribution minimizes the number of edges in the role-based graph model. Each user-permission set is first considered as a possible role. Then, the algorithm identifies two roles such that the optimization criteria (the number of edges + the number of roles) is improved. The improvement is made by merge or split operations based on the set relationship between two selected permission sets. This process is performed iteratively until improvement is no longer possible. However, the number of iterations needed to reach the most optimal criteria is not clear.

Another study has defined several variations of the Role Mining Problem (RMP) [33]. The authors use the matrix form to denote all kinds of role mining problems. Also, they map the RMP to Minimum 


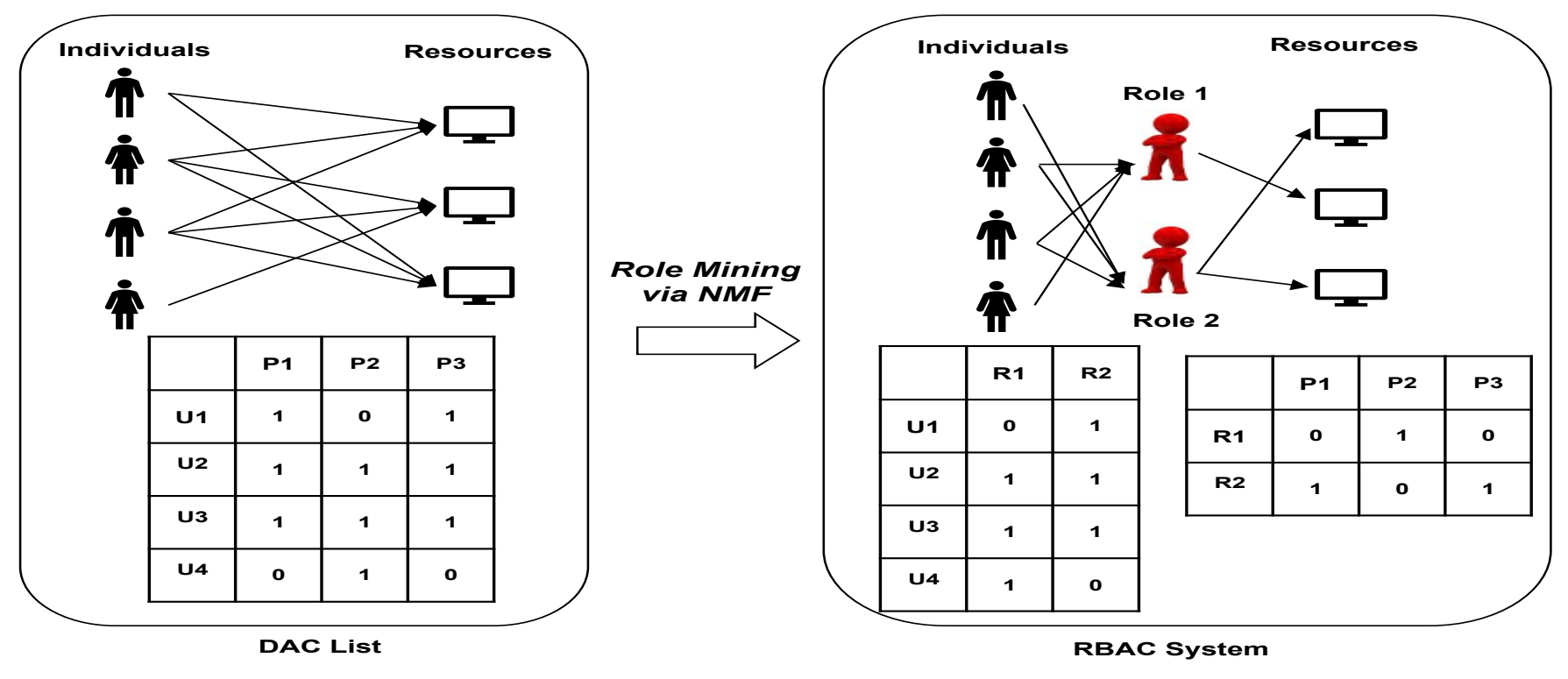

Figure 1: Overview of DAC to RBAC Migration via Matrix Decomposition

Tiling Problem and Discrete Basis Problem by showing that RMP and $\delta-R M P$ are NP-complete problems. Nonetheless, this approach suffers from high time complexity.

Alternatively, authors in [10] propose a heuristic algorithm to identify the minimum number of roles [10]. In this approach, the algorithm selects a user $u$ and finds a pair $\langle U(u) ; P(u)>$ as a role in each step, where $U(u)$ refers to all users who possess the same permissions as those of $u$, and $P(u)$ denotes the set of permissions of a user $u$. All user-permission assignments between the $U(u)$ and $P(u)$ are then deleted; thus only the remaining user-permission assignments are allowed to pass to the next iteration.

Zhang et al. generate a set of candidate roles using frequent pattern mining and then employ partial ordering to create a role hierarchy [35]. In this approach, there are no limitations on either the number of permissions that can be assigned to any specific role or the number of roles allocated for any given user. Nonetheless, this approach creates a high number of candidate permission sets, which can negatively impact the throughput efficiency of the algorithm.

Other methods in role mining problem include HierarchicalMiner and similarity-based hierarchical role mining. The former is a formal analysis-based approach that discovers roles with their semantic meanings [22]. Roles with semantic meanings seem to be easier to maintain in practice. The authors study the role mining problem in two cases. Once when the user-permission relation is the only available information and once with the user-attribute information also available. This paper uses WSC (Weighted Structural Complexity) as an evaluation measure to evaluate the resulted role set. In similarity-based hierarchical role mining approach, a reduced concept lattice is initially generated followed by pruning the existing lattice and selecting the new RBAC state [31]. The new RBAC state has the minimum perturbation in comparison with the existing RBAC state. However, the proposed algorithm is not evaluated by real data.
Alternatively, an optimal role mining problem can be achieved by transforming the role mining problem into a constraint satisfaction problem [11]. This transformation allows for discovering the optimal RBAC state based on customized optimization metrics. Notably, the time complexity of the approach is exponential to the size of users and permissions. Lack of scalability is a caveat of this approach.

In an attempt to reduce the complexity of the role mining process, Colantonio et al. propose a methodology composed of three steps [7]. The authors initially define a threshold that is used to identify unstable user-permission relations. Roles are then extracted only from stable assignments; thus the administration cost is minimized. The quality of the extracted roles, however, is ignored in this method.

Alternatively, another role mining approach considers weights for permissions based on their importance [21]. More specifically, weights are computed according to the similarity between users and permissions, which are then utilized for the extraction of the roles.

Bai et al. [2] propose a framework for role mining based on the multi-domain information named RMMDI. This framework uses information in multi-domains, including network domain, digital domain, and physical domain, to discover the relationships between users and permissions.

Abolfathi et al. [1] propose a scalable and context-aware role mining algorithm based on graph embedding and unsupervised feature learning. To capture and learn the structural similarity of permissions, they use an efficient random walk sampling-based approach results in extracting roles that are meaningful in the context of the organization. Firstly, they convert the access control list to a bipartite graph called "user-permission graph" and then use clustering to find subsets of similar nodes and group them together as potential roles. However, to address scalability, instead of performing clustering on the original graph, they employ a graph 
embedding method to represent data in a new low-dimensional space. Then, a clustering algorithm is applied in a low-dimensional space to extract candidate roles. Finally, a prune and refine algorithm is developed to verify the final roles and cover the corner cases. Although this novel approach works well, it only can reconstruct $67 \%$ of the original role set.

Another category of works includes machine learning approaches. Prominent ones are clustering-based methods, where the whole RBAC construction of user-role-permission is generated by the clustering algorithms, and the suggested set of roles is given as the output. For example, Schlegelmilch in [27] proposes a method called ORCA that uses the hierarchical clustering method to investigate the user-permissions relation and then combines the ORCA tools to illustrate the clustering results in a graphical form. Initially, this algorithm clusters the users who have the largest same permissions together. Then, it merges and updates the permissions clusters according to the maximum number of common users to create a new cluster. ORCA repeats the process until no user has permission in any two clusters. In this method, the permissions in the generated role sets cannot overlap. Since, in typical use cases, permissions are often shared among roles, this drawback significantly limits the accuracy of the model.

Occasionally, the input $U P$ data in certain cases may contain noise, which should be cleared to prevent error migration from the input data to the resulting RBAC system. Subsequently, role mining can be performed. In line with this, a previous study has recommended a two-step process for mining roles associated with noisy data [24]. In this approach, matrix decomposition techniques are first employed to clean the input data. Next, role mining is done. Moreover, several context-aware RBAC models have been proposed in the field to accommodate the context-awareness in access control management; [6, 9, 12, 25, 30].

In summary, existing approaches suffer from the trade-off between optimality and scalability. More scalable approaches are based on heuristics and thus are not optimal, while optimal approaches like [11] suffer from lack of scalability.

\subsection{Matrix Factorization}

In this section, we explain some popular matrix factorization methods. Matrix factorization methods such as Principal Component Analysis [13], Singular Value Decomposition (SVD) [4] or NonNegative Matrix Factorization (NMF) [3] are popular in data mining and machine learning. The Principal Component Analysis (PCA) is one of the powerful linear dimension reduction methods. PCA decreases the dimension of the data by finding a few orthogonal linear combinations of the original variables with the largest variance [13]. PCA is simple to implement, but extracted components are hard to interpret. Singular value decomposition (SVD) is another approach of matrix factorization. The technique is usually used where eigenvectors are of interest. SVD decomposes the original matrix into three constituent matrices. In particular, the SVD algorithm factorizes a matrix into one matrix with orthogonal columns and one with orthogonal rows (along with a diagonal matrix, which contains the relative importance of each factor). It is usually used to remove redundant features from the dataset [15]. It uses the concept of eigenvalues and eigenvectors to determine those three matrices.
In particular, the SVD allows us to discover the same kind of information as the eigen decomposition. NMF has become a widely-used algorithm for the analysis of high-dimensional data as it efficiently extracts sparse and meaningful features from a set of non-negative data vectors $[3,18]$. The reason that NMF is so popular is its ability to extract interpretable factors. NMF is described in more detail in Section 2.

\section{PROPOSED METHODOLOGY}

We propose three criteria for a perfect role mining technique:

- Accuracy: it must divide the existing binary user-permission matrix into two new assignments, i.e. user-role and rolepermission such that the original user-permission is fully reconstructed from their multiplication; i.e., all original permissions are assigned to users, and no extra permission is assigned to any user.

- Optimality: the number of resulting roles, $r$, must be minimum.

- Scalability: the role mining process must be scalable (reasonable mining time) to large data sets with millions of permissions.

Since the role mining problem is NP-complete, achieving all above goals at the same time is challenging. In this paper, we propose a scalable role mining approach that achieves perfect accuracy and generates an optimal number of roles for small datasets and a near-optimal number of roles for large ones. Next, we formally define these objectives and present our methodologies for realizing them.

\subsection{Problem Definition}

Definition 1. (Optimal and Accurate Matrix Decomposition): Given a matrix $U P \in\{0,1\}^{n \times m}$, the optimal and accurate matrix decomposition derives two matrices $U R \in\{0,1\}^{n \times r}$ and $R P \in\{0,1\}^{r \times m}$ such that $r$ is minimum and reconstruction rate is 1.0.

In this work, we assume the input DAC assignments are sound and complete (noiseless) which basically means and the user-permission assignments in the input $U P$ soundly and completely represents the set of all desirable accesses of the system.

\subsection{Proposed Procedure}

The input user-permission matrix, which consists of a number of users $(n)$ and permissions $(m)$, captures the permission assignment for each user in an organization. To discover the existing roles in the input user-permission matrix, the matrix should be factorized as $U P \approx U R \times R P$ through the NMF procedure. To this end, it is required to first assign the desired value for the number of roles, which is indicated by $r$. This creates a mapping of the assignments to various roles e.g. $\left\{R_{1}, \ldots, R_{r}\right\}$ in the matrix, with each role holding a varying degree of membership as determined by the factorization process demonstrated below. 
$\left[\begin{array}{ccc}u p_{11} & \cdots & u p_{1 n} \\ \vdots & \ddots & \vdots \\ \vdots & \ddots & \vdots \\ u p_{n 1} & \cdots & u p_{n m}\end{array}\right] \approx\left[\begin{array}{ccc}u r_{11} & \cdots & u r_{1 r} \\ \vdots & \vdots & \ddots \\ \vdots & \vdots & \ddots \\ u r_{n 1} & \cdots & u r_{n r}\end{array}\right] \times\left[\begin{array}{ccc}r p_{11} & \cdots & r p_{1 m} \\ \vdots & \vdots & \ddots \\ r p_{r 1} & \cdots & r p_{r m}\end{array}\right]$

In the above equation, a value $u r_{i j} \in U R$ ( with $\left.0 \leq u r_{i j} \leq 1\right)$ indicates the degree of membership of the role $r_{j}$ to the user $u_{i}$. Likewise, value $r p_{i j} \in R P$ ( with $0 \leq r p_{i j} \leq 1$ ) implies the degree of membership of the permission $p_{j}$ in $U P$ to the role $r_{i}$. Notably, in the RBAC model, binary values for user-roles and role-permissions are desirable rather than the relative real values. Obtaining binary values can be achieved by applying acceptable thresholds (e.g. $t_{1}, t_{2}$ below) on the degree of membership for roles and permissions, which are demonstrated as $u r_{i j}^{n e w}$ and $r p_{i j}^{n e w}$ in Equation 6. In Section 5 , we discuss how to choose the values for $t_{1}$ and $t_{2}$.

$$
u r_{i j}^{n e w}=\left\{\begin{array}{ll}
1 & \text { if } u r_{i j} \geq t_{1} \\
0 & \text { if } u r_{i j}<t_{1}
\end{array} \quad r p_{i j}^{n e w}= \begin{cases}1 & \text { if } r p_{i j} \geq t_{2} \\
0 & \text { if } r p_{i j}<t_{2}\end{cases}\right.
$$

To examine the efficiency of NMF in detecting latent factors in the dataset, we reconstruct $U P^{n e w}$ by multiplying $U R^{n e w}$ and $R P^{n e w}$ matrices. We then perform an element by element comparison between the input $U P$ and $U P^{n e w}$ to determine the success rate of NMF in decomposing binary user-permission matrix into user-role and role-permission relations. In most cases, it covers up to $99 \%$ of original data, and in the worst case up to $96 \%$. The time complexity of the algorithm is $O(\mathrm{~nm})$.

Importantly, selecting an optimal value for the decomposition rank $(r)$ is critical for a proper role mining. This can be accomplished in several ways. For instance, the decomposition process can be started with a very low rank, which is then gradually increased until we reach an $r$ value with the highest reconstruction rate. Alternatively, one can achieve an optimal $r$ value by repeating NMF several times per rank and then calculate how similar or stable the results are.

As another approach, one may evaluate the ability of decomposition for recovering unknown values and how it can generalize for unseen data using cross-validation. To this end, a small portion of data can be held out and considered as unknown data or test data. The $r$ value that can provide the best approximation of the missing values on test data should be selected as the optimal rank for decomposition. One can repeat this procedure leaving out a portion of elements one at a time and sum up the prediction errors over the test data. However, this can be computationally costly because the NMF has to be repeated for each left-out elements.

In our prepared datasets, we know the number of existing original roles prior to role mining. However, the number of roles is not known in a real-world scenario which makes finding the optimal $r$ value a challenging task. Thus, to estimate the optimal $r$ value, we employ Elbow and Silhouette score analysis methods [14].

The Elbow method seeks to minimize the total within-cluster sum of squares (WSS) as a function of the number of clusters intuitively. The value for $r$ will then be selected as the number of clusters when adding another cluster does not significantly improve the clustering accuracy [32]. The Silhouette measure determines how well each point lies within its cluster. In other words, it measures the quality of a clustering method by doing an investigation on the separation distance between the output clusters. A high average Silhouette score indicates that the clustering has performed well [26].

Moreover, it is critically important the selected $r$ value satisfy the Equation 7. In line with this, we applied the optimal $r$ values obtained by Elbow and Silhouette approaches, into the Equation 7 as outlined below:

$$
((n+m) \times r)<(n \times m)
$$

Once the $r$ value satisfies the Equation 7, we repeat the NMF several times per rank with different numbers close to the selected $r$ values. Each entered value might have a slight positive or negative effect on the accuracy of the system. Accordingly, we select the $r$ value with the best reconstruction rate.

From the security perspective, it is critical to ensure that migration from DAC to RBAC does not compromise the security within an organization. For instance, if an extracted role assigns a user with extra permissions, it may result in adverse security side-effects. On the other hand, an extracted role should not deprive users of permissions that are required to complete a job, as this would lead to the user's failure in performing the assigned duties. Indeed, each user must have the same permissions in the RBAC model as those in the original DAC model. To this end, we propose a refinement step (Algorithm 3) that covers all corner cases such that the built $U P$ matrix by $U R^{n e w}$ and $R P^{n e w}$ is complete as the input $U P$ matrix. In this study, we only observed the under-assignments, and no over-assignments occurred in our experiments. But further investigation is required to ensure that the over-assignments never occurs, which is deferred to our future work. In this step, we first identify the minimal number of roles containing the maximum permission coverage from the role set $\mathrm{R}$ which is the output of algorithm 2. Notably, some roles may have extra or over-assigned permissions for a user $u$, which should be removed from the downstream analysis. Finally, we identify the permissions that are not yet covered by any existing roles and assign them to a new role.

\section{EXPERIMENTAL EVALUATION}

We conduct an experimental study to evaluate the efficiency of our proposed approach. To evaluate the accuracy of a role mining approach, it is ideal to perform all experimental analyses using real-world user-permission data. However, obtaining such data is extremely difficult, especially those containing complete RBAC states. Hence, most role mining algorithms have utilized synthetic user-permission data as input for evaluation [23]. Likewise, we prepare our input datasets based on a template developed by a previous study at Stony Brook University [29]. This template generates an RBAC model in a typical university data system for studying security analysis in the role-based access control model. The template includes the typical roles and permissions settings present in a university system. Thus, it closely reflects the structure and the access control policy structure of a real environment. 

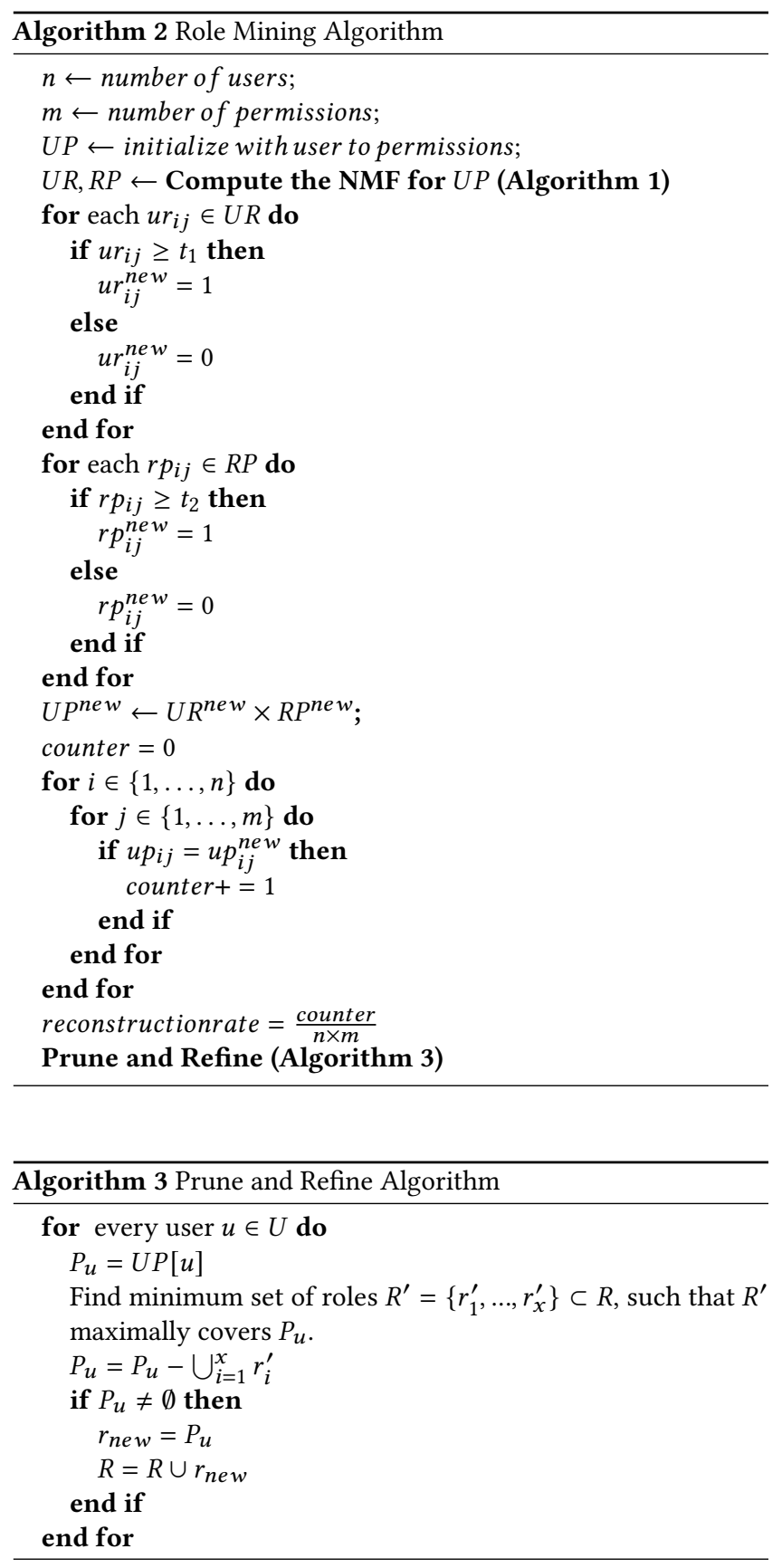

\subsection{Data Preparation}

In this study, we perform our experiments and analyses on five different datasets that are demonstrated in Table 1 . The datasets are prepared using the procedure shown in Algorithm 4. First, we define a set of roles based on the above-mentioned template. This is followed by creating a number of users that are then randomly assigned to each role, with a specified maximum number of users for any given role. We then set the user-permissions according to the roles assigned to each user in the study.
Table 1: Characteristics of Datasets

\begin{tabular}{||l|c|c|c|c||}
\hline Dataset & $\mid$ Users $\mid$ & $\mid$ Permissions $\mid$ & $|\mathrm{UP}|$ & Density \\
\hline \hline Dataset1 & 1000 & 37 & 8140 & 0.22 \\
Dataset2 & 5000 & 37 & 70300 & 0.38 \\
Dataset3 & 8000 & 91 & 29120 & 0.04 \\
Dataset4 & 27027 & 1000 & 305181 & 0.011 \\
Dataset5 & 108054 & 4000 & 56188080 & 0.13 \\
\hline
\end{tabular}

\subsection{Experimental Settings}

We have implemented our test bed in python and C\#. The experiments were performed on a Core-i7 $3.60 \mathrm{GHz}$ Intel(R) CPU with 8 gigabytes of memory.

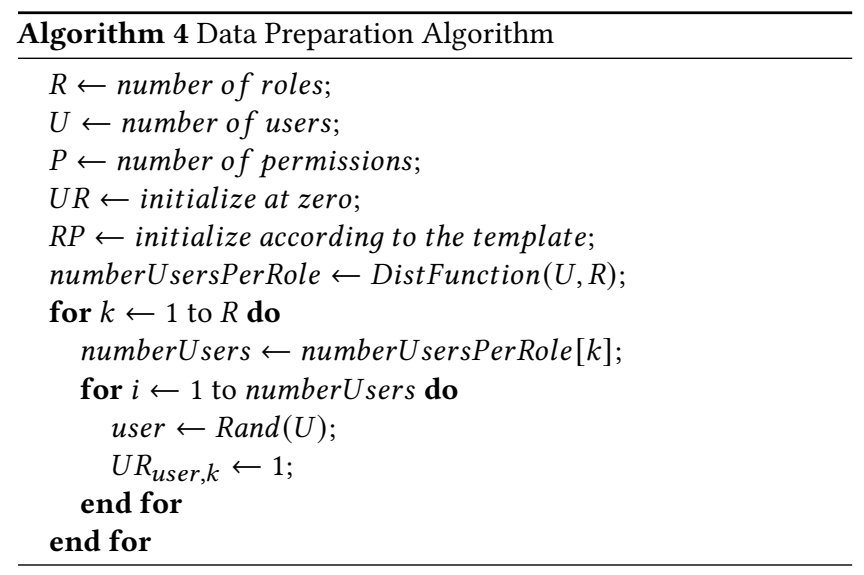

\subsection{Experimental Result Analysis}

We initially performed role mining analysis on Dataset1, i.e. the small dataset, and demonstrated that our NMF approach has $100 \%$ reconstruction rate (see Table 2). In other words, for the small dataset, our NMF approach performs perfectly well and there are no under- or over-assignment of permissions. A great performance was also observed for the larger datasets 2, 3, 4, and 5, where the reconstruction rates reached $96.5 \%, 97 \%, 99 \%$, and $96 \%$, respectively. Note that these are the construction rates that are achieved after applying the NMF decomposition. While an above $80 \%$ coverage is considered sufficient in some literature works [24], our aim is to ensure a $100 \%$ reconstruction rate to reach perfect accuracy. To achieve this, the heuristic prune-and-refine algorithm updates the user-role and role-permission assignments to ensure that a perfect reconstruction rate is achieved as the final output.

Table 2: Role Mining Results for the Prepared Datasets

\begin{tabular}{||c|c|c|c||}
\hline $\mid$ Users $\mid$ & $\mid$ Permissions $\mid$ & $|\mathrm{UP}|$ & Accuracy (\%) \\
\hline \hline 1000 & 37 & 8140 & 100 \\
5000 & 37 & 70300 & 96.5 \\
8000 & 91 & 29120 & 97 \\
27027 & 1000 & 305181 & 99 \\
108054 & 4000 & 56188080 & 96 \\
\hline
\end{tabular}


Figure 2 illustrates the number of original roles used for preparing the datasets against the number of extracted roles. The number of original roles and extracted roles (i.e. rank decomposition) are indicated by red and green bars, respectively. Depicted by blue bars are the number of final extracted roles, which are calculated after applying the prune and refining algorithm. Notably, the number of extracted roles among different datasets is close to the number of original roles, indicating our approach is very close to the optimal solution. More specifically, the number of extracted roles is identical to the number of original roles for Dataset1, i.e. the small-scale dataset. Relative to the original roles, the rate of extracted roles increased to $11 \%, 4 \%, 5 \%$, and $10.87 \%$ for datasets $2,3,4$, and 5 , respectively, which is still acceptable for this medium- to large-scale datasets.

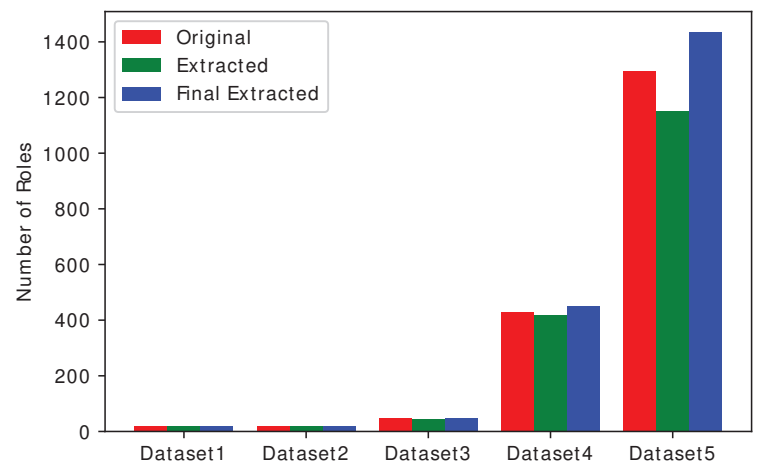

Figure 2: Comparison of Initial vs. Extracted Number of Roles

We select optimal values for rank decomposition utilizing the Elbow and Silhouette methods. As an example, we provide an Elbow graph for Dataset2 that is shown in Figure 3. Then, we use the Equation 7 to make sure that the selected $r$ values are correct. For instance, using the Elbow method, we choose an $r$ value of 18 for the Dataset2 that contains 5000 users $(n)$ and 37 permissions $(m)$. Likewise, the same $r$ value, i.e. $r=18$, satisfies Equation 7 when $n=5000$, and $m=37$.

The execution times for all five datasets are shown in Figure 4. As illustrated, the processing time increases as the dataset size increases. The $y$-axis is on the logarithmic scale. Execution time increases exponentially with the input size, but it is still acceptable for very large datasets. For example, for the largest dataset, the execution time is less than three hours.

Also, we calculate the error ratio for different values of $r$. The error ratio for Dataset3 is presented in Figure 5. We can see that the minimum error occurs for $r=418$.

Thus far, we have explained how a non-negative matrix factorization can be employed to extract user-to-role and role-to-permission relations in the RBAC context. Next, we discuss how we addressed the issue we encountered.

To find the optimal values for thresholds, we conduct an experiment to investigate how the accuracy of the NMF decomposition

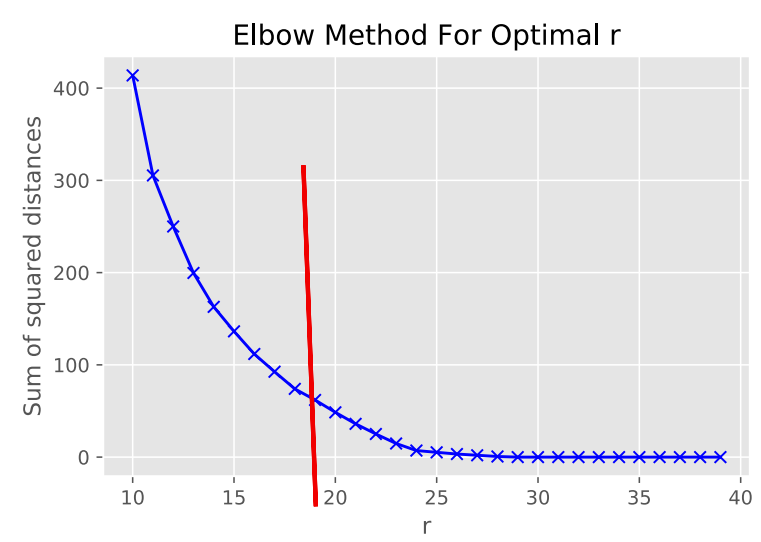

Figure 3: K-means Clustering SSE vs. Decomposition Rank for Dataset2

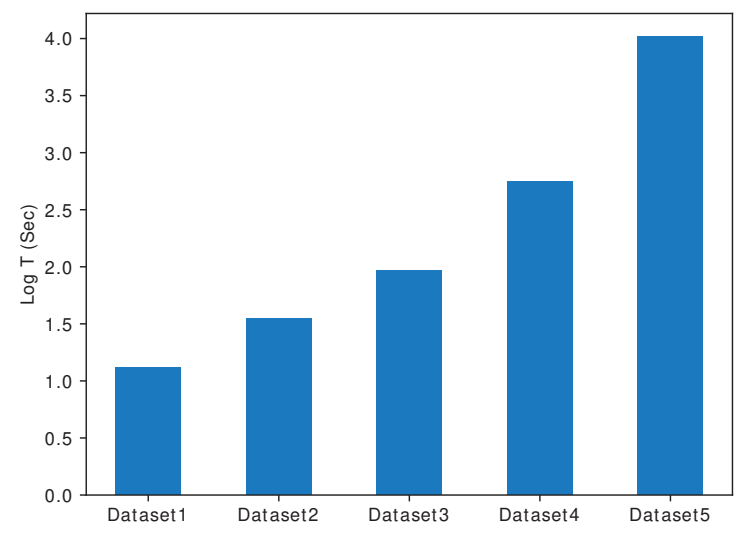

Figure 4: Runtime on Five Dataset (s)

changes by altering the threshold. As demonstrated in Figure 6, increasing the threshold values does not cause any significant variance in the accuracy. Thus, it seems that threshold optimization is not required, as its changes would not affect the accuracy of our role mining approach in any significant way. Accordingly, we use threshold $t_{1}=0.5$ and $t_{2}=0.5$, in which if the degree of membership for a role to a given user is greater than or equal to 0.5 , the role is assigned to that user. Similarly, if the membership degree for permission to a role is greater than or equal to 0.5 , that permission is assigned to the role.

\section{CONCLUSION AND FUTURE WORK}

In this paper, we propose a novel approach for extracting the latent factor concept behind the user-permission data. The latent factor hidden in the user-permission (DAC list) is called "role". The key idea of the approach is to mine user-role and role-permission relations from the existing user-permission information in a scalable and optimal manner. We use the NMF technique to decompose 


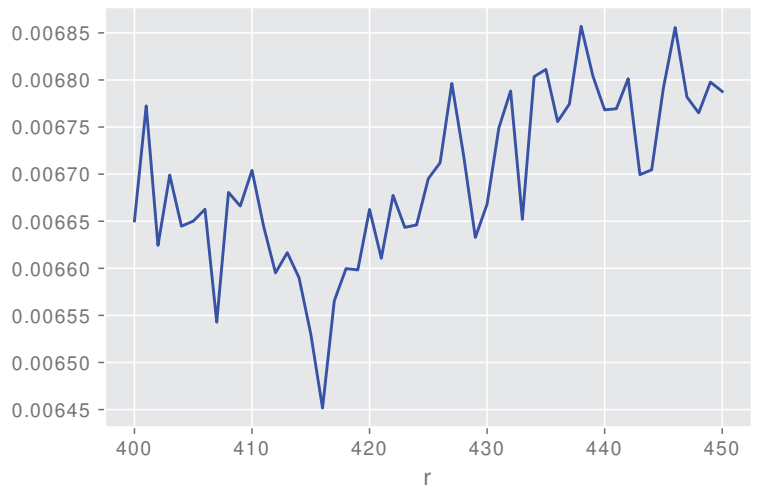

Figure 5: Error vs. Decomposition Rank (r)

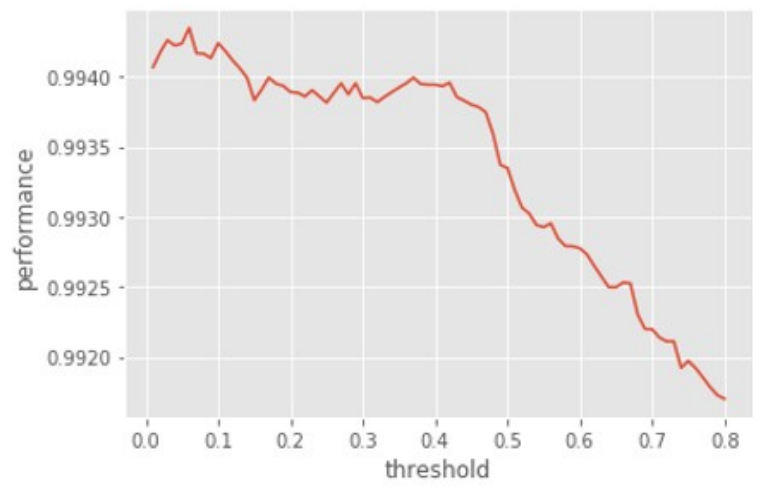

Figure 6: Parameter Sensitivity: Threshold Values

the input binary matrix into two low-rank binary matrices. Experiments on five different data sets demonstrate that the NMF can extract the hidden concept effectively and reasonably.

For future work, we will move in two directions. The first direction is to meet the real needs in practice by considering the restrictions, including hierarchies in our model. Second, we will try different variations of matrix factorization techniques or clustering methods such as autoencoder which can be a suitable fit for the role mining problem.

\section{REFERENCES}

[1] Masoumeh Abolfathi, Zohreh Raghebi, Jafar Haadi Jafarian, and Farnoush BanaeiKashani. 2020. An Accurate and Scalable Role Mining Algorithm based on Graph Embedding and Unsupervised Feature Learning. In Proceedings of the 53rd Hawaii International Conference on System Sciences.

[2] Wei Bai, Zhisong Pan, Shize Guo, and Zhe Chen. 2019. RMMDI: a novel framework for role mining based on the multi-domain information. Security and Communication Networks 2019 (2019).

[3] Michael W. Berry, Murray Browne, Amy N. Langville, V. Paul Pauca, and Robert J. Plemmons. 2007. Algorithms and applications for approximate nonnegative matrix factorization. Computational Statistics \& Data Analysis 52, 1 (2007), 155 173. https://doi.org/10.1016/j.csda.2006.11.006

[4] Dheeraj Bokde, Sheetal Girase, and Debajyoti Mukhopadhyay. 2015. Matrix Factorization Model in Collaborative Filtering Algorithms: A Survey. Procedia Computer Science 49 (2015), 136 - 146. https://doi.org/10.1016/j.procs.2015.04. 237 Proceedings of 4th International Conference on Advances in Computing, Communication and Control (ICAC3'15)

[5] Axel Buecker, Werner Filip, Jaime Cordoba Palacios, Andy Parker, et al. 2009. Identity management design guide with IBM Tivoli Identity Manager. IBM Redbooks.

[6] Jung Hwan Choi, Hyunsu Jang, and Young Ik Eom. 2010. CA-RBAC: Context Aware RBAC Scheme in Ubiquitous Computing Environments. Fournal of Information Science \& Engineering 26, 5 (2010).

[7] Alessandro Colantonio, Roberto Di Pietro, Alberto Ocello, and Nino Vincenzo Verde. 2010. Taming role mining complexity in RBAC. Computers \& Security 29, 5 (2010), 548-564.

[8] Andrej Čopar, Blaž Zupan, and Marinka Zitnik. 2019. Fast optimization of nonnegative matrix tri-factorization. PloS one 14, 6 (2019).

[9] Michael J Covington, Wende Long, Srividhya Srinivasan, Anind K Dev, Mustaque Ahamad, and Gregory D Abowd. 2001. Securing context-aware applications using environment roles. In Proceedings of the sixth ACM symposium on Access control models and technologies. 10-20.

[10] Alina Ene, William G. Horne, Nikola Milosavljevic, Prasad Rao, Robert Schreiber, and Robert Endre Tarjan. 2008. Fast exact and heuristic methods for role minimization problems. In 13th ACM Symposium on Access Control Models and Technologies, SACMAT 2008, Estes Park, CO, USA, fune 11-13, 2008, Proceedings. 1-10. https://doi.org/10.1145/1377836.1377838

[11] Jafar Haadi Jafarian, Hassan Takabi, Hakim Touati, Ehsan Hesamifard, and Mohamed Shehab. 2015. Towards a General Framework for Optimal Role Mining: A Constraint Satisfaction Approach. In Proceedings of the 20th ACM Symposium on Access Control Models and Technologies, Vienna, Austria, June 1-3, 2015. 211-220. https://doi.org/10.1145/2752952.2752975

[12] Kangsoo Jung and Seog Park. 2013. Context-aware role based access control using user relationship. International fournal of Computer Theory and Engineering 5, 3 (2013), 533.

[13] Nandakishore Kambhatla and Todd K Leen. 1997 Dimension Reduction by Local Principal Component Analysis. Neural Computation 9, 7 (1997), 1493-1516. https://doi.org/10.1162/neco.1997.9.7.1493 arXiv:https://doi.org/10.1162/neco.1997.9.7.1493

[14] Trupti M Kodinariya and Prashant R Makwana. 2013. Review on determining number of Cluster in K-Means Clustering. International fournal 1, 6 (2013), 90-95.

[15] Bang Le, Kien Nguyen, and Ruck Thawonmas. 2015. Bounded-SVD: A Matrix Factorization Method with Bound Constraints for Recommender Systems. Proceedings - 2015 2nd International Conference on Emerging Information Technology and Engineering Solutions, EITES 201524 (04 2015), 23-26. https: //doi.org/10.1109/EITES.2015.10

[16] Daniel D Lee and H Sebastian Seung. 1999. Learning the parts of objects by non-negative matrix factorization. Nature 401, 6755 (1999), 788-791.

[17] Daniel D Lee and H Sebastian Seung. 2001. Algorithms for non-negative matrix factorization. In Advances in neural information processing systems. 556-562.

[18] Daniel D. Lee and H. Sebastian Seung. 2001. Algorithms for Non-negative Matrix Factorization. In Advances in Neural Information Processing Systems 13, T. K. Leen, T. G. Dietterich, and V. Tresp (Eds.). MIT Press, 556-562. http://papers.nips.cc/ paper/1861-algorithms-for-non-negative-matrix-factorization.pdf

[19] Haibing Lu, Jaideep Vaidya, and Vijayalakshmi Atluri. 2008. Optimal Boolean Matrix Decomposition: Application to Role Engineering. In Proceedings of the 24th International Conference on Data Engineering, ICDE 2008, April 7-12, 2008, Cancún, Mexico. 297-306. https://doi.org/10.1109/ICDE.2008.4497438

[20] Haibing Lu, Jaideep Vaidya, and Vijayalakshmi Atluri. 2014. An optimization framework for role mining. Journal of Computer Security 22, 1 (2014), 1-31.

[21] Xiaopu Ma, Ruixuan Li, and Zhengding Lu. 2010. Role mining based on weights. In Proceedings of the 15th ACM symposium on Access control models and technologies. 65-74.

[22] Ian Molloy, Hong Chen, Tiancheng Li, Qihua Wang, Ninghui Li, Elisa Bertino, Seraphin Calo, and Jorge Lobo. 2008. Mining roles with semantic meanings. In Proceedings of the 13th ACM symposium on Access control models and technologies. 
$21-30$.

[23] Ian Molloy, Ninghui Li, Tiancheng Li, Ziqing Mao, Qihua Wang, and Jorge Lobo 2009. Evaluating role mining algorithms. In Proceedings of the 14th ACM symposium on Access control models and technologies. 95-104.

[24] Ian Molloy, Ninghui Li, Yuan Qi, Jorge Lobo, and Luke Dickens. 2010. Mining roles with noisy data. In Proceedings of the 15th ACM symposium on Access control models and technologies. 45-54.

[25] Seon-Ho Park, Young-Ju Han, and Tai-Myoung Chung. 2006. Context-role based access control for context-aware application. In International Conference on High Performance Computing and Communications. Springer, 572-580.

[26] Peter J Rousseeuw. 1987. Silhouettes: a graphical aid to the interpretation and validation of cluster analysis. Journal of computational and applied mathematics 20 (1987), 53-65.

[27] Jürgen Schlegelmilch and Ulrike Steffens. 2005. Role mining with ORCA. In 10th ACM Symposium on Access Control Models and Technologies, SACMAT 2005, Stockholm, Sweden, June 1-3, 2005, Proceedings. 168-176. https://doi.org/10.1145/ 1063979.1064008

[28] Farial Shahnaz, Michael W Berry, V Paul Pauca, and Robert J Plemmons. 2006 Document clustering using nonnegative matrix factorization. Information Processing \& Management 42, 2 (2006), 373-386.
[29] Scott D Stoller, Ping Yang, C R Ramakrishnan, and Mikhail I Gofman. 2007. Efficient policy analysis for administrative role based access control. In Proceedings of the 14th ACM conference on Computer and communications security. 445-455.

[30] Muhammad Nabeel Tahir. 2007. C-RBAC: Contextual role-based access control model. Ubiquitous Computing and Communication fournal 2, 3 (2007), 67-74.

[31] Hassan Takabi and James B. D. Joshi. 2010. StateMiner: an efficient similaritybased approach for optimal mining of role hierarchy. In 15th ACM Symposium on Access Control Models and Technologies, SACMAT 2010, Pittsburgh, Pennsylvania, USA, fune 9-11, 2010, Proceedings. 55-64. https://doi.org/10.1145/1809842.1809853

[32] Robert L Thorndike. 1953. Who belongs in the family. In Psychometrika. Citeseer.

[33] Jaideep Vaidya, Vijayalakshmi Atluri, and Qi Guo. 2007. The role mining problem: finding a minimal descriptive set of roles. In Proceedings of the 12th ACM symposium on Access control models and technologies. 175-184.

[34] Jaideep Vaidya, Vijayalakshmi Atluri, and Janice Warner. 2006. "RoleMiner: mining roles using subset enumeration". In Proceedings of the 13th ACM Conference on Computer and Communications Security, CCS 2006, Alexandria, VA, USA, Ioctober 30 - November 3, 2006. 144-153. https://doi.org/10.1145/1180405.1180424

[35] Dana Zhang, Kotagiri Ramamohanarao, Tim Ebringer, and Trevor Yann. 2008. Permission set mining: Discovering practical and useful roles. In 2008 Annual Computer Security Applications Conference (ACSAC). IEEE, 247-256. 